\title{
Scaled Effective Solvent Method for Predicting the Equilibrium Ensemble of Structures with Analysis of Thermodynamic Properties of Amorphous Polyethylene Glycol-Water Mixtures
}

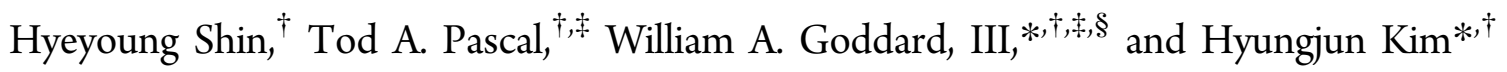 \\ ${ }^{\dagger}$ Graduate School of EEWS and ${ }^{\S}$ World Class University Professor, Korea Advanced Institute of Technology (KAIST), Daejeon, \\ Korea \\ ${ }^{\ddagger}$ Materials and Process Simulation Center, California Institute of Technology, Pasadena, California 91125, United States
}

Supporting Information

\begin{abstract}
Water-soluble polymers such as polyethylene glycol (PEG) are critical components of industrial processes ranging from drug delivery to water purification. However, the understanding of the microscopic structure of these polymers in water and of the thermodynamics of the mixtures is limited because available experimental techniques (such as SLS and SANS) give little information about conformations and provide even the radius of gyration only in the dilute limit $(<\sim 5$ wt \% PEG). Computer simulations employing Monte Carlo (MC) and molecular dynamics (MD) techniques can provide an atomistic molecular structure; however, such approaches have difficulties in predicting the equilibrium polymer configurations of highmolecular-weight polymers at normal densities and in obtaining entropies and free energies directly from the MD. Here, we develop the scaled effective solvent (SES) method to predict the equilibrium ensemble of polymer

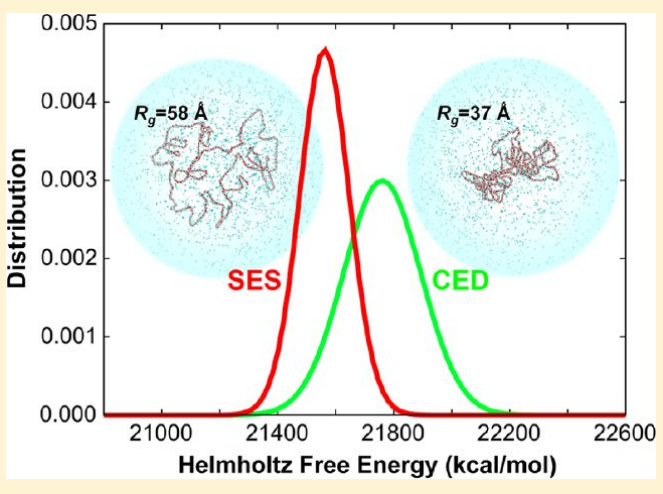
configurations, which we illustrate for the case of a $20 \mathrm{kDa}$ PEG (455 monomers) at a 25 wt \% PEG aqueous solution (3339 waters per PEG chain). We evaluate the free energy and entropy of the members of this ensemble including explicit water, validating that it leads to average sizes $\left(R_{\mathrm{g}}\right)$ observed experimentally and that all members of the ensemble have favorable free energies. With the SES method validated to provide well-equilibrated polymer chains in water, it should be useful for predicting ensembles of polymer chains in polymer melts and in solvents.
\end{abstract}

\section{INTRODUCTION}

Understanding the nature of polymer-solvent interactions is quite important in designing optimal systems for industrial applications ranging from drug delivery to water purification and fuel cell electrolytes. Such design is hampered by limitations in experimental characterization, making it most difficult to develop an atomistic understanding beyond the limits of very dilute solutions. In order to provide this atomistic understanding, we are developing new methodologies, which we illustrate here for the case of polyethylene glycol (PEG) water mixtures, a watersoluble polymer with wide-ranging applications. Indeed, interactions of PEG with water resemble that of a protein ${ }^{1}$ so that such studies provide insights into the more complicated interactions and function of proteins and enzymes. In particular, the substantial ability of PEG mixtures to absorb and transport water molecules ${ }^{2}$ is of great importance in commercial and technological applications, such as pharmaceutical drugs delivery, $^{3-7}$ cosmetics, ${ }^{8,9}$ and functioning fuel cells. ${ }^{10}$

To determine the atomic-level structure of PEG solution and the interaction of PEG with water molecules, a number of experimental methods have been applied, including dynamic light scattering (DLS), ${ }^{11-13}$ static light scattering (SLS), ${ }^{12,14,15}$ Raman spectroscopy, ${ }^{16,17}$ nuclear magnetic resonance
(NMR) ${ }^{18-20}$ infrared spectroscopy (IR), ${ }^{19}$ and small-angle neutron scattering (SANS). ${ }^{21,22}$ These methods provide various levels of macroscopic structural information, such as the overall size (radius of gyration, $R_{\mathrm{g}}$ ) of the PEG chains and local clustering. However, such measurements are indirect and ensemble-averaged, providing little information about atomistic structures. In addition, structural characterizations based on SLS or SANS assume the Guinier condition that is valid only in the dilute regime $(<\sim 5 \mathrm{wt} \%)$, where polymer-polymer interactions are negligible. Consequently, experimental characterizations of polymer structures in the semidilute regime $(20-35 \mathrm{wt} \%)^{23}$ are rare. Other important properties such as osmotic behavior of these mixtures are difficult to predict because the contribution of the second virial term to osmotic pressure is significant in this semidilute regime.

In contrast, molecular dynamics (MD) simulations can provide atomistic structures and thermodynamics for interpretation of experimental studies. However, the complexity of sampling the polymer configuration space for $20 \mathrm{kDa}$ PEG

Received: October 22, 2012

Revised: December 20, 2012

Published: December 20, 2012 
(PEG-20 kDa) with its 455 monomer units (1365 independent torsions) is huge and becomes even more difficult when considering the water or other solvent explicitly. Thus, previous MD studies of PEG structures in water have generally been limited to low-molecular-weight polymers $\left(<\sim 1 \mathrm{kDa}^{24-27}\right.$ or 22 monomer units). For practical applications, the most interesting sizes for PEGs are much larger, with molecular weights of 40$100 \mathrm{kDa}$ commonly used in biomedical and biotechnical applications. In this regime, experimental limitations have not resolved such questions as whether the PEGs in water are aggregated under various conditions. ${ }^{11,28}$

We report here the scaled effective solvent (SES) computational method designed to sample a sufficiently wide range of polymer conformations in solution in which we can be confident that the properties of fully equilibrated polymer chains are captured. In the SES method, we start with isolated chains but include the effect of the solvent and other polymer chains being ignored by scaling the intrachain nonbonding interactions. This mimics the effect of solvent and other molecular chains in denser polymer systems, which counterbalance the attractiveness of intrachain interactions. We then combine this SES procedure with a new first-principles-based force field developed here to describe the intermolecular interaction between water and PEG to predict the atomistic structures of PEG-20 kDa for the most interesting PEG concentration in an aqueous solution of $25 \mathrm{wt} \%$ (semidilute), a regime where it is difficult to extract structural data from experiment.

We then analyze the entropy and free energy of these mixtures using the two-phase thermodynamic (2PT) method. ${ }^{29}$ We find an equilibrium ensemble of polymer chains with a wide range of sizes, $R_{\mathrm{g}}=34.86-78.77 \AA$, each with similar free energies, indicating that this distribution of chains represents the equilibrium ensemble. The final thermodynamically averaged size is $R_{\mathrm{g}}=53.98 \AA$ for $25 \%$ PEG in water at $300 \mathrm{~K}$. This is in agreement with experiment in the dilute limit, $R_{\mathrm{g}}=65 \AA$, and with the $R_{\mathrm{g}}=56.68 \AA$, assuming a model of segments freely jointed at the $\mathrm{O}$ atoms with a fixed $\mathrm{C}-\mathrm{O}-\mathrm{C}$ angle.

\section{COMPUTATIONAL DETAILS}

2.1. First-Principles-Based Force Field. To obtain an accurate description of water molecules interacting with the PEG

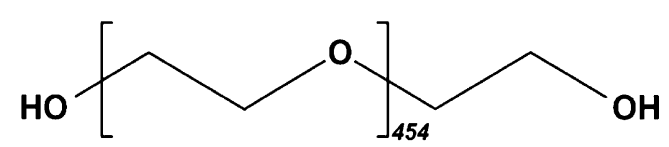

Figure 1. Chemical structure of a PEG-20 kDa chain end-capped with hydroxyl $(-\mathrm{OH})$ groups, which consists of 455 repeating monomers.

backbone, we developed a new force field (FF) by modifying the DREIDING $^{30}$ generic FF to reproduce the quantum mechanics (QM) energies and structures for the hydrogen bond interaction of water molecules with the ether oxygen atoms of the PEG backbone. Using dimethyl ether (DME) to represent a repeating unit of PEG interacting with $\mathrm{H}_{2} \mathrm{O}$, we determined these interactions using the M06-2X density functional ${ }^{31}$ with the Dunning correlation-corrected triple- $\zeta$ basis sets (cc-PVTZ $++{ }^{32}$ in Jaguar 7.6 program $^{33}$ ). Figure S1a (Supporting Information (SI)) shows the optimized structure from QM calculations (global minimum configuration) along with two other structures optimized while constraining the point group symmetry to remain $C_{2 v}$ (Figure $S 1 b$ and S1c, SI). We fitted these QM results to a Morse-type off-diagonal van der Waal's term and a
DREIDING-like explicit three-body hydrogen bonding term for the interactions of the ether oxygen atoms in the PEG backbone with the hydrogens in water molecule. Here, we used the 12-10 Lennard-Jones functional term for the radial term.

Figures S1 and S2 (SI) show that the FF describes well the intermolecular interactions from $\mathrm{QM}$, including the global minimum configuration (Figure S1a, SI). The optimum FF parameters are listed in Table S1 (SI). The partial charge distributions $(q)$ (Table S2, SI) of the PEG molecule were determined to fit the electrostatic potential (ESP) charges derived from QM calculation for the 1,2-ethanediol molecule (which represents the PEG model with a repeating unit; $n=1$ ).

For the water-water interaction, we used the flexible threecentered (F3C) water model ${ }^{34}$ with a $-0.82 \mathrm{e}^{-}$partial charge on oxygen $\left(q_{\mathrm{O}(\text { wat }}\right)$ and a $0.41 \mathrm{e}^{-}$partial charge on hydrogen $\left(q_{\mathrm{H}(\text { wat }}\right)$. The spring constant for the $\mathrm{OH}$ bond is $500 \mathrm{kcal} / \mathrm{mol} /$ $\AA^{2}$, with an equilibrium bond length of $1.0 \AA$, and the spring constant for $\mathrm{HOH}$ angle is $120 \mathrm{kcal} / \mathrm{mol} / \mathrm{rad}^{2}$, with an equilibrium angle of $109.47^{\circ}$.

2.2. MD Simulations. As a prototypical PEG, we considered the $20 \mathrm{kDa}$ molecular weight linear polymer, consisting of 455 repeating monomers (terminating both end groups with hydroxyl, $-\mathrm{OH}$ ), as shown in Figure 1.

As a representative case for the semidilute regime, we focused on the detailed structures and energetics of PEG in aqueous solution with a polymer concentration of $25 \mathrm{wt} \%$. The simulation cell for a $25 \mathrm{wt} \%$ PEG aqueous solution consisted of a single PEG-20 kDa chain ( 3188 atoms) solvated by 3339 water molecules, leading to 13205 atoms in the simulation cell. We carried out MD simulations using the large-scale atomistic modeling massively parallelized simulation (LAMMPS) code. ${ }^{35}$ Here, the isothermal damping constant for Nosé-Hoover NVT calculations was $100 \mathrm{fs}$, and the isobaric damping constant for Nosé-Hoover NPT calculations was 1000 fs.

\section{RESULTS USING THE SOLVENT-INCLUDED COHESIVE ENERGY DENSITY (SI-CED) METHOD}

Our system with the 1365 independent torsions leads to $\sim 3^{1365} \sim 10^{651}$ possible conformers, not counting the solvent. For

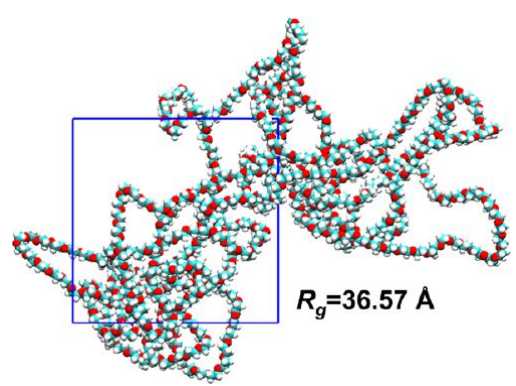

Figure 2. Atomistic structure of a PEG-20 kDa with $R_{\mathrm{g}}=36.57 \AA$ in a PEG aqueous solution with 25 wt \% of PEG built using SI-CED at the final full density, $\rho=1.05 \mathrm{~g} / \mathrm{cm}^{3}$ (comparable to the experimentally measured density, $\left.\rho^{\exp }=1.04 \mathrm{~g} / \mathrm{cm}^{3}\right)$. White, red, and green atoms are hydrogen, oxygen, and carbon atoms, respectively. The periodic box is shown as a blue solid line. The equilibrated structure by SI-CED is partially aggregated due to the self-solvation effect resulting from substantial intrachain interaction during vacuum dynamics (no water) and has much higher energy than the sampled structures with our new procedure (see Figure 9). Therefore, we conclude that the SI-CED is not a proper procedure for sampling high-molecular-weight polymer structures dissolved in a solvent. 


\section{Last 5 ns equilibrated structures $\left(f=0.3\right.$ and $\left.\rho=0.01 \mathrm{~g} / \mathrm{cm}^{3}\right)$}
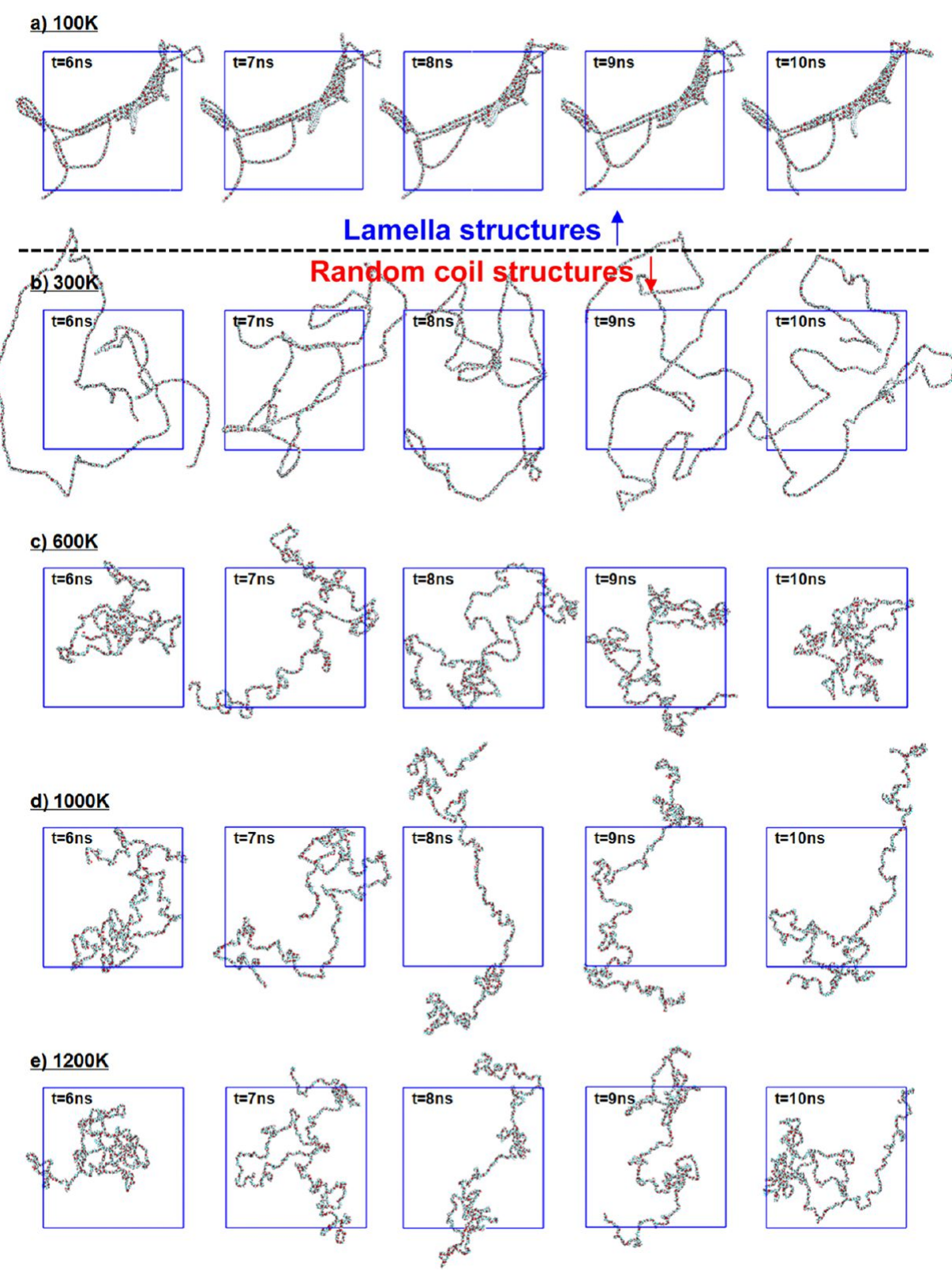

Figure 3. The $f=0.3$ SES case. Structures of a PEG-20 kDa chain with $\rho=0.01 \mathrm{~g} / \mathrm{cm}^{3}$ sampled over the last $5 \mathrm{~ns} N V T$ dynamics at $T=(\mathrm{a}) 100,(\mathrm{~b}) 300$, (c) 600, (d) 1000, and (e) $1200 \mathrm{~K}$. Over the temperature range from 300 to $1200 \mathrm{~K}$, the last $5 \mathrm{~ns}$ of equilibrated PEG-20 kDa conformations all show random coil-like behavior in which the backbone of the polymer moves randomly in three-dimensional space, while at $200 \mathrm{~K}$ and below, the PEG structures maintain a lamellar shape. White, red, and green atoms are hydrogen, oxygen, and carbon atoms, respectively. The periodic box is shown as a blue solid line.

such a dense polymer melt or solution, $\mathrm{MD}$ is far too slow to allow the system to equilibrate fully in practical time scales. Consequently, Monte Carlo (MC) or simulated annealing (SA) methods are generally used in such systems. ${ }^{36,37}$ However, for long polymer chains at normal density, the MC growth process can lead to nonuniform conformations due to exclusion effects as the full length of the growing polymer is reached. To overcome this limitation, Goddard and co-workers ${ }^{38}$ developed the cohesive energy density (CED) method, which involves

(1) growing the polymer at half of the expected density, $0.5 \rho$,

(2) compression of the simulation cell from $0.5 \rho$ to $1.1 \rho$ with five quench-anneal cycles [300-706 K ( twice the Flory temperature; $\left.353 \mathrm{~K}^{39}\right)$ ] using NVT, and
(3) relaxation of the final structure from step 2 using NPT MD for $0.25 \mathrm{~ns}$ to obtain the final structure.

Indeed, CED has been applied to predict Hildebrand solubility parameters, ${ }^{40-42}$ the structures of complex polymers such as Nafion ${ }^{43}$ and Dendrimer-modified electrolytes, ${ }^{44}$ and thermodynamics ${ }^{45}$ using MD simulation.

A complication with the CED method is how to incorporate the solvent. In previous studies ${ }^{43,46}$ the amount of water was small, and it was sufficient to add them in at the 50\% density level, right after the "polymer growth step in vacuum". This is denoted as the solvent-included CED; SI-CED method.

An example of the structures built from SI-CED is shown in Figure 2 (25 wt \% PEG at the final full density, $\rho=1.05 \mathrm{~g} / \mathrm{cm}^{3}$ ). 
Last $5 \mathrm{~ns}$ equilibrated structures $\left(f=1.0\right.$ and $\left.\rho=0.01 \mathrm{~g} / \mathrm{cm}^{3}\right)$

a) $100 \mathrm{~K}$
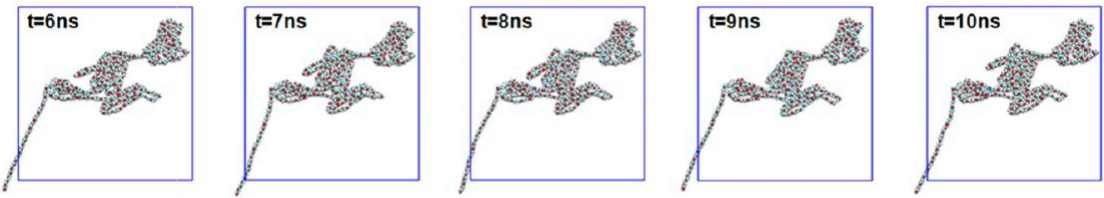

b) $300 \mathrm{~K}$
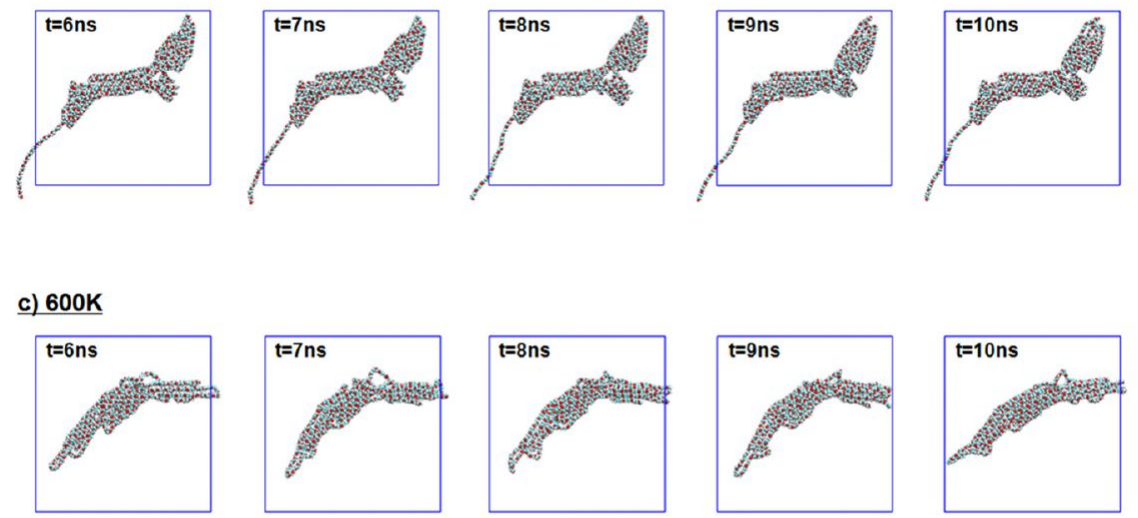

\section{Lamella structures $\uparrow$}
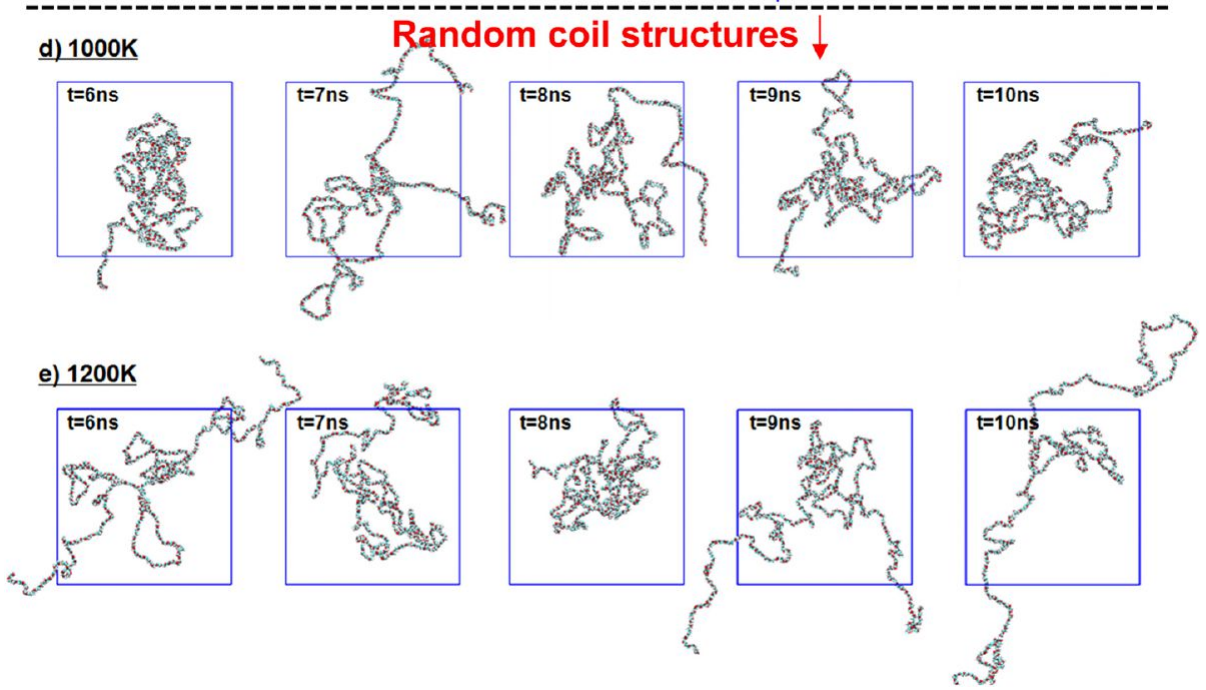

Figure 4. The $f=1.0$ SES case. Structures of a PEG- $20 \mathrm{kDa}$ chain with $\rho=0.01 \mathrm{~g} / \mathrm{cm}^{3}$ sampled over the last $5 \mathrm{~ns} N V T$ dynamics at $T=(\mathrm{a}) 100$, (b) 300 , (c) 600, (d) 1000, and (e) $1200 \mathrm{~K}$. Over the temperature range from 900 to $1200 \mathrm{~K}$, the last $5 \mathrm{~ns}$ of equilibrated PEG-20 kDa conformations all show random coil-like behavior in which the backbone of the polymer moves randomly in three-dimensional space, while at $800 \mathrm{~K}$ and below, the PEG structures maintain a lamellar shape. White, red, and green atoms are hydrogen, oxygen, and carbon atoms, respectively. The periodic box is shown as a blue solid line.

We see that for $25 \mathrm{wt} \%$ PEG in water, SI-CED leads to PEG chains containing nonuniform polymer globules. This occurs because of the strong intrachain interaction (i.e., self-solvation effect) during the polymer growth procedure in vacuum prior to water insertion. These partially globular conformations from SICED bias the sampling toward the initial self-solvated conformation, which survives even after compression/expansion cycles using the temperature annealing procedures in CED.

On the basis of these results, we concluded that the SI-CED strategy is not appropriate for a broad sampling of highmolecular-weight polymer structures immersed in a solvation box. In fact, we show below that the PEG-water mixture sampled and equilibrated with SI-CED leads to a much higher energy than the structures built with our new procedure and that the size of the SI-CED chain is at the small size limit of our final distributions (vide infra).

\section{SES-CED METHOD}

4.1. SES Concept. In order to develop an efficient but unbiased sampling of polymer configurations for high degrees of solvation, we developed the SES-CED method. SES-CED samples the polymer chain conformations of an isolated chain but with reduced nonbonding interactions (van der Waals, hydrogen bonds, and electrostatic) scaled by a factor $f$ to account 


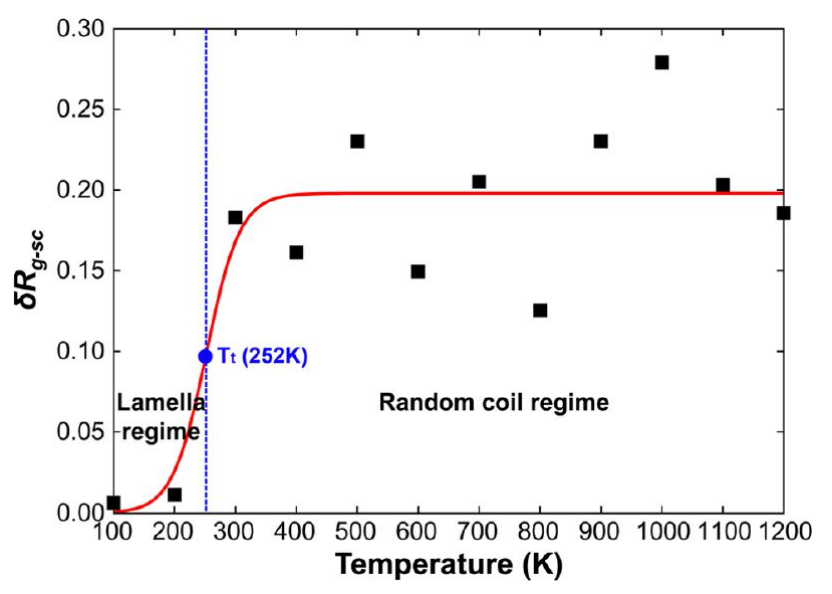

Figure 5. The scaled standard deviation of $R_{\mathrm{g}}, \delta R_{\mathrm{g}-\mathrm{sc}}=$ (standard deviation of $\left.R_{\mathrm{g}}\right) /\left(\right.$ average $R_{\mathrm{g}}$ ), for $f=0.3$ over the last 5 ns $N V T$ dynamics for each temperature ranging from 100 to $1200 \mathrm{~K}$ using a large simulation cell with $\rho=0.01 \mathrm{~g} / \mathrm{cm}^{3}$. We find that low temperature leads to a globular or lamella structures with small $\delta R_{\text {g-sc }}(<\sim 0.01)$, and high temperature leads to a random coil (R-coil) structure with $\delta R_{\text {g-sc }}>$ $\sim 0.13$. At the highest temperature, the value of $\delta R_{\mathrm{g}-\mathrm{sc}}$ is similar to the value expected for an infinite Gaussian coil, 0.26 . To determine a precise $\theta$ temperature $\left(T_{\mathrm{t}}\right)$ to serve as the boundary between these two regimes, we used the hyperbolic tangent function, $\delta R_{\mathrm{g}-\mathrm{sc}}(T)=a\left[\tanh \left\{\left(T-T_{\mathrm{t}}\right) /\right.\right.$ b\} +1$]$, where $T_{\mathrm{t}}$ is indicated by the blue point.

for the solvent being ignored (the effective solvent). The idea is that in the real polymer system, there are attractive interactions of the polymer chain with solvent and other polymer chains ignored in the single-chain vacuum representation, while the intrachain interactions are included explicitly. This case $(f=1.0)$ favors globule or lamella formation. Using a reduced $f$ for the isolated single-chain calculations reduces the bias of the intrachain interactions, allowing chain structures to more closely resemble the actual chain conformations in a polymer melt or a waterpolymer mixture while still allowing rapid and efficient sampling of the polymer chain over all possible conformations and sampling the multiple basins in the potential energy surface.

4.2. Phase Diagram of Polymer Chain Conformations (As a Function of $f$ and $T$ ). In order to choose a proper scaling factor, $f$, we performed MD simulations on the isolated polymer chain to sample a wide range of PEG chain sizes in a large simulation box $\left(140 \times 140 \times 140 \AA^{3}\right)$ with $\rho=0.01 \mathrm{~g} / \mathrm{cm}^{3}$. Here, we varied $f$ from 0.1 to 1.0 and carried out NVT MD for $10 \mathrm{~ns}$ at each temperature of $100-1200 \mathrm{~K}$. The $R_{\mathrm{g}}$ values for the last $5 \mathrm{~ns}$ of each case are shown in Figure S3 (SI). Note that we use the term $R_{\mathrm{g}}$ to be $\left\langle R_{\mathrm{g}}{ }^{2}\right\rangle^{1 / 2}$. Snapshots of the conformations for $f=0.3$ and 1.0 are shown in Figures 3 and 4, respectively, and snapshots for the other cases are shown in Figures S4-S6 (SI). These results show that there is a clear transition temperature $\left(T_{\mathrm{t}}\right)$ at $\sim 300 \mathrm{~K}$ for $f=0.3$ and $\sim 1000 \mathrm{~K}$ for $f=1.0$. We denote this transition temperature as the coil-globule or $\theta$ temperature. Defining the scaled fluctuations in the radius of gyration as

$$
\delta R_{\mathrm{g}-\mathrm{sc}}=\frac{\left(\text { standard deviation of } R_{\mathrm{g}}\right)}{\left(\text { average } R_{\mathrm{g}}\right)}
$$

we find the following:

-Below $T_{\mathrm{t}}$ intrachain attraction dominates, leading to polymer chain conformations that adopt lamella-like chain structures that change little with time $\left(\delta R_{\mathrm{g}-\mathrm{sc}}<\sim 0.04\right)$. This is the poor solvent condition, in which the polymer chains favor globular a) Relative Standard Deviation $R_{g}(\AA)$

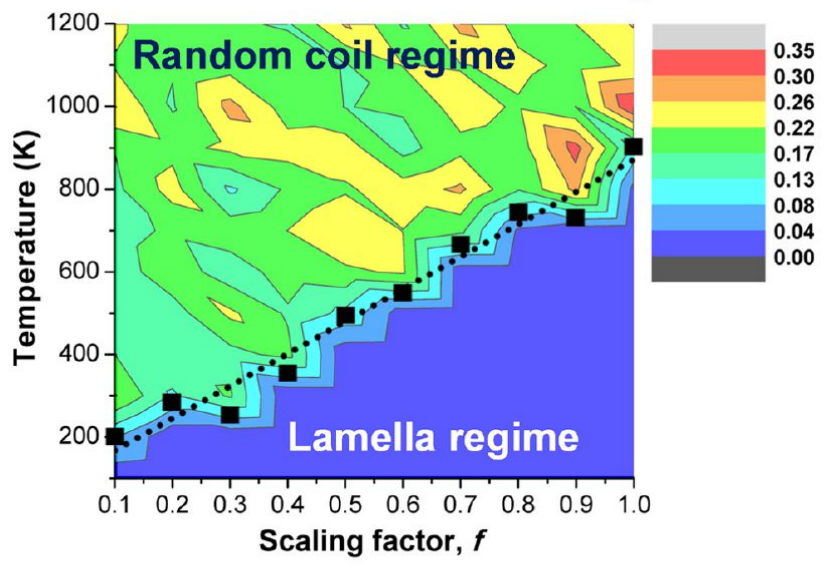

\section{b) Average $R_{g}(\AA)$}

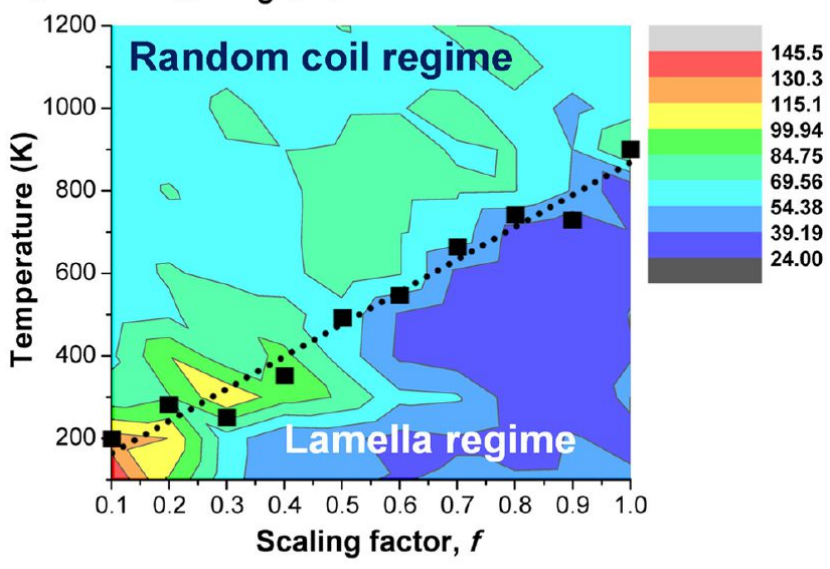

c) Maximum $R_{g}(\AA)$

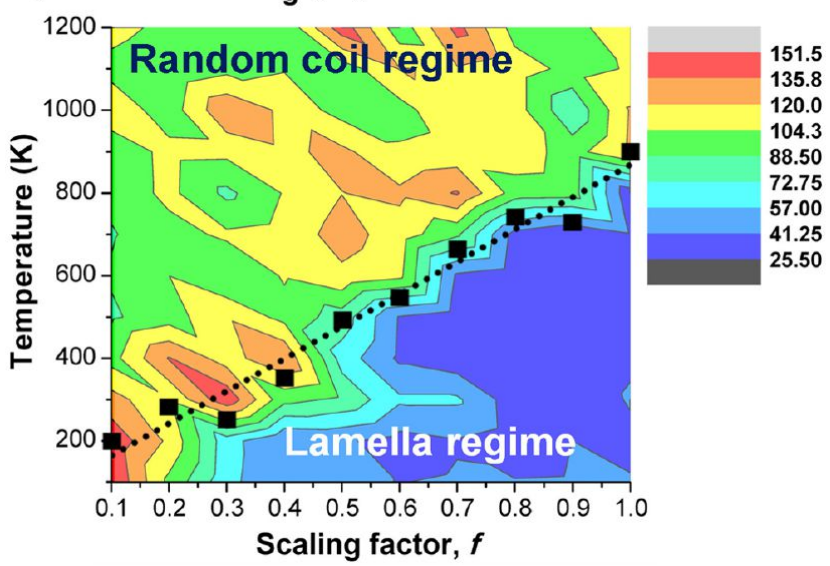

Figure 6. Phase diagram for conformations of an isolated PEG chain showing the dramatic change in properties above the $\theta$ temperature characterized by rapidly fluctuating random coil conformations and below the $\theta$ temperature characterized by frozen lamella conformations. (a) Relative standard deviation of $R_{g}$, b) average value of $R_{g}$, and (c) maximum value of $R_{\mathrm{g}}$. The relative standard deviation of $R_{\mathrm{g}}, \delta R_{\mathrm{g}-\mathrm{sc}}$ is the normal standard deviation of $R_{\mathrm{g}}$ divided by the average $R_{\mathrm{g}}$. Color bars indicate the magnitudes of $R_{\mathrm{g}}$. The black squares with a least-squares fit indicate the $\theta$ temperature $\left(T_{\mathrm{t}}\right)$ separating the two regimes, which are quite distinctive.

conformations to minimize polymer-solvent contacts and maximize contacts between polymer segments. 
a)

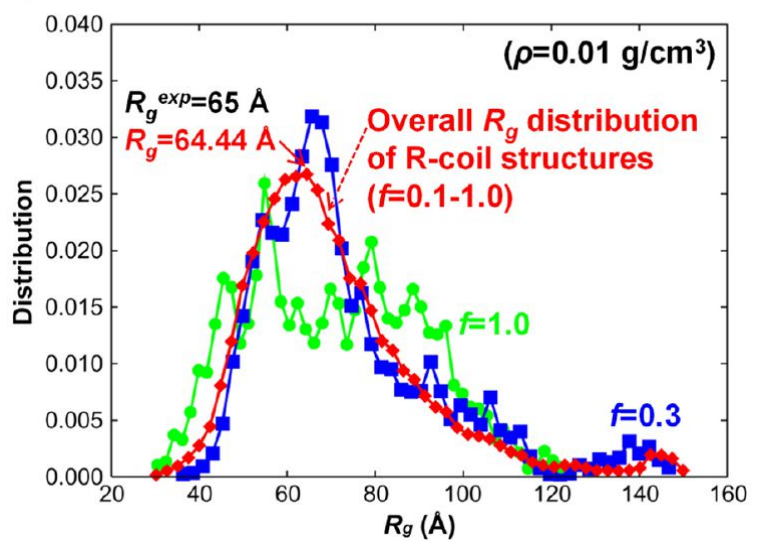

b)

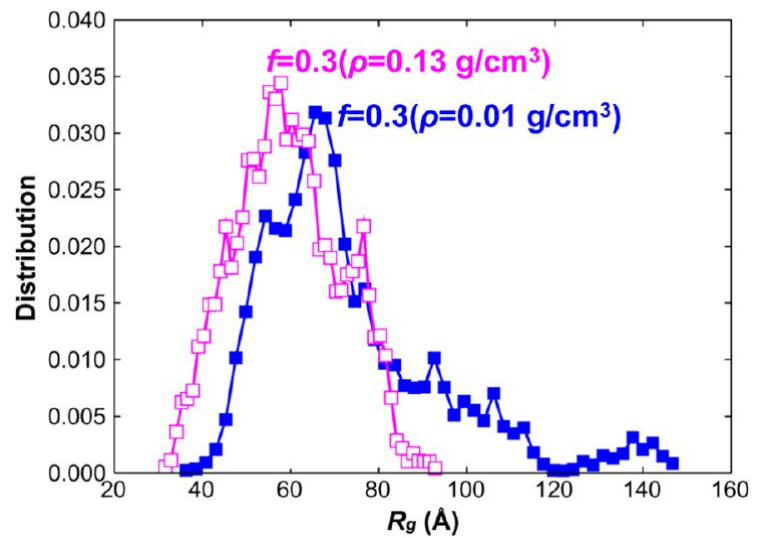

Figure 7. (a) Red line: the overall $R_{\mathrm{g}}$ distribution obtained over the temperature range from 100 to $1200 \mathrm{~K}$ for scaling factors $(f)$ (from $f=0.1$ to 1.0 ) using a large simulation box $\left(140 \times 140 \times 140 \AA^{3}\right)$ with $\rho=0.01 \mathrm{~g} / \mathrm{cm}^{3}$. Blue and green lines: $R_{\mathrm{g}}$ distributions obtained by using $f=0.3$ (blue) or 1.0 (green). (b) Comparison of the $R_{\mathrm{g}}$ distribution obtained by using $f=0.3$ and varying the temperature in a $63.52 \times 63.52 \times 63.52 \AA^{3}$ simulation box, leading a density of $\rho=0.13 \mathrm{~g} / \mathrm{cm}^{3}$ (if we insert $75 \mathrm{wt} \%$ water, then the total density should be $0.52 \mathrm{~g} / \mathrm{cm}^{3}$, which is half of the density expected from experiment $\rho^{\exp }=1.04 \mathrm{~g} / \mathrm{cm}^{3}$ ) (magenta line) with the $R_{\mathrm{g}}$ distribution obtained by using $f=0.3$ and varying the temperature in a large simulation box with $\rho=0.01 \mathrm{~g} / \mathrm{cm}^{3}$ (blue line).

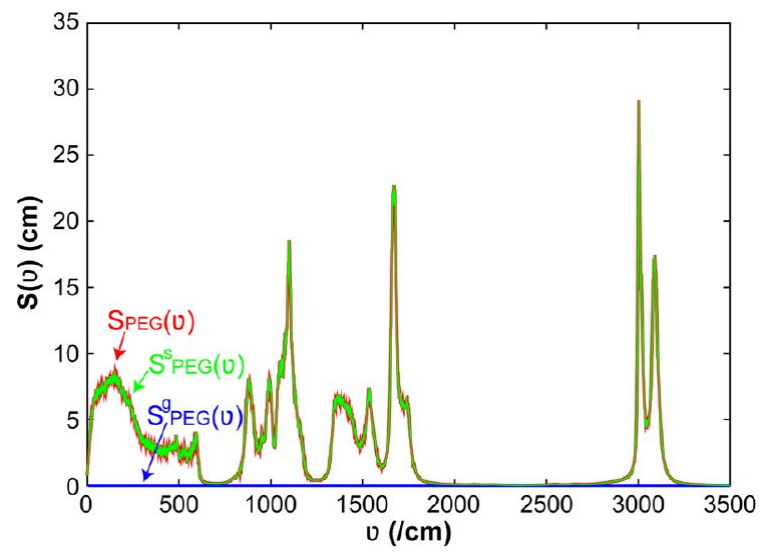

Figure 8. Starting with the PEG chain having $R_{\mathrm{g}}=55.67 \AA$, the DoS for the PEG from the Fourier transform of the velocity autocorrelation function by the $2 \mathrm{PT}$ analysis at $300 \mathrm{~K}$ for the $25 \mathrm{wt} \% \mathrm{PEG}$ aqueous solution. The red line is the DoS of the PEG, $S_{\mathrm{PEG}}(v)$. Blue and green lines are gas-like $S_{\mathrm{PEG}}^{\mathrm{g}}(v)$ and solid-like $S_{\mathrm{PEG}}^{\mathrm{s}}(v)$ components of $S_{\mathrm{PEG}}(v)$, respectively. Most of the diffusional contribution is from the water dynamics. The SI shows the total DoS and that of the water for several cases.

-Above $T_{\mathrm{t}}$ thermal (entropic) effects dominate, leading to fluctuating random coil structures. This is the good solvent condition, in which the polymer chains favor a random coil structure that maximizes configurational entropy, leading to extended conformations.

To determine the $T_{\mathrm{t}}$ for each scaling factor, we consider how $\delta R_{\mathrm{g}-\mathrm{sc}}$ changes with temperature. These are shown in Figure 5 for the case of $f=0.3$ and in Figure S7 (SI) for the other cases. Here, we see that at the lowest temperatures, the lamella or globule region leads to very small fluctuations, $\delta R_{\mathrm{g}-\mathrm{sc}}<\sim 0.04$, while the highest temperatures lead to $\delta R_{\mathrm{g}-\mathrm{sc}}=0.19-0.26$, close to the infinite chain limit for a Gaussian coil of $\delta R_{\mathrm{g}-\mathrm{sc}}=0.26$. (for details, see Table S3 and S4, SI).

To define the $\theta$ temperature $\left(T_{\mathrm{t}}\right)$ forming the boundary between these two regimes, we used the hyperbolic tangent function, $\delta R_{\mathrm{g}-\mathrm{sc}}(T)=a\left[\tanh \left\{\left(T-T_{\mathrm{t}}\right) / b\right\}+1\right]$. For $f=0.3$, Figure 5 shows that this leads to $T_{\mathrm{t}}=252 \mathrm{~K}$.
Figure 6 shows the phase diagram for the PEG conformations as a function of $f$ and temperature, where $T_{\mathrm{t}}$ is indicated by the black squares. The lamella structure regime shows characteristics such as small $\delta R_{\mathrm{g}-\mathrm{sc}}$ small maximum $R_{\mathrm{g}}$, and small average $R_{\mathrm{g}}$ due to very limited movements dominated by the strong intrachain interactions. In contrast, the random coil (R-coil) structure regime exhibits opposite characteristics such as large $\delta R_{\text {g-so }}$ approaching the limit of 0.26 for infinite Gaussian chains, large maximum $R_{\mathrm{g}}$, and moderate average $R_{\mathrm{g}}$.

4.3. Choosing the Initial Ensemble of Polymer Chain Conformations. For a good solvent such as water, we expect that moderately dilute solutions of PEG at $300 \mathrm{~K}$ should lead to R-coil structures; therefore, we considered only these cases in choosing the initial ensemble of polymer chain conformations.

Figure 7a (red solid line) shows the overall $R_{\mathrm{g}}$ distribution obtained in a large simulation box $\left(140 \times 140 \times 140 \AA^{3}\right)$ with $\rho=$ $0.01 \mathrm{~g} / \mathrm{cm}^{3}$ by averaging over all temperatures (from 100 to 1200 $\mathrm{K})$ and scaling factors $(f=0.1-1.0)$ but including only cases in the R-coil regime (we note that temperature-dependent $R_{\mathrm{g}}$ distributions are shown in Figure S8, SI). This leads to a broad range of PEG structures (red solid line) with $\Delta R_{\mathrm{g}} \approx 120 \AA$, and the most probable value of $R_{\mathrm{g}}$ is $64.44 \AA$.

Also shown in Figure $7 \mathrm{a}$ are the $R_{\mathrm{g}}$ distributions obtained in a large simulation box $\left(140 \times 140 \times 140 \AA^{3}\right)$ with $\rho=0.01 \mathrm{~g} / \mathrm{cm}^{3}$ by using $f=0.3$ (blue solid line) and 1.0 (green solid line) for all temperatures from $T_{\mathrm{t}}$ to $1200 \mathrm{~K}$. The single case of $f=0.3$ (blue) leads to an $R_{\mathrm{g}}$ distribution similar to the overall $R_{\mathrm{g}}$ distribution (red). Indeed, the peak of the distribution for the case of $f=0.3$ is at $R_{\mathrm{g}}=65.58 \AA$, very close to the peak of the overall distribution, $R_{\mathrm{g}}=64.44 \AA$. Therefore, we conclude that MD simulations using only $f=0.3$ but varying the temperature up to $\sim 1200 \mathrm{~K}$ lead to a distribution of conformations appropriate for spanning the conformations of a PEG chain in solution. This can be compared to studies ${ }^{47}$ by Jang and Goddard on linear and cyclic polyethylene, where $f=0.28-0.35$ led to $\theta$ temperatures in good agreement with experimental measurements. [In addition, $f$ $=0.1$ can be another option because it also leads to similar range of $R_{\mathrm{g}}$ as in the case of $f=0.3$ (see Figure S9, SI).]

As discussed above, sampling of PEG structures using SES dynamics with $f=0.3$ within the large simulation box with $\rho=$ 

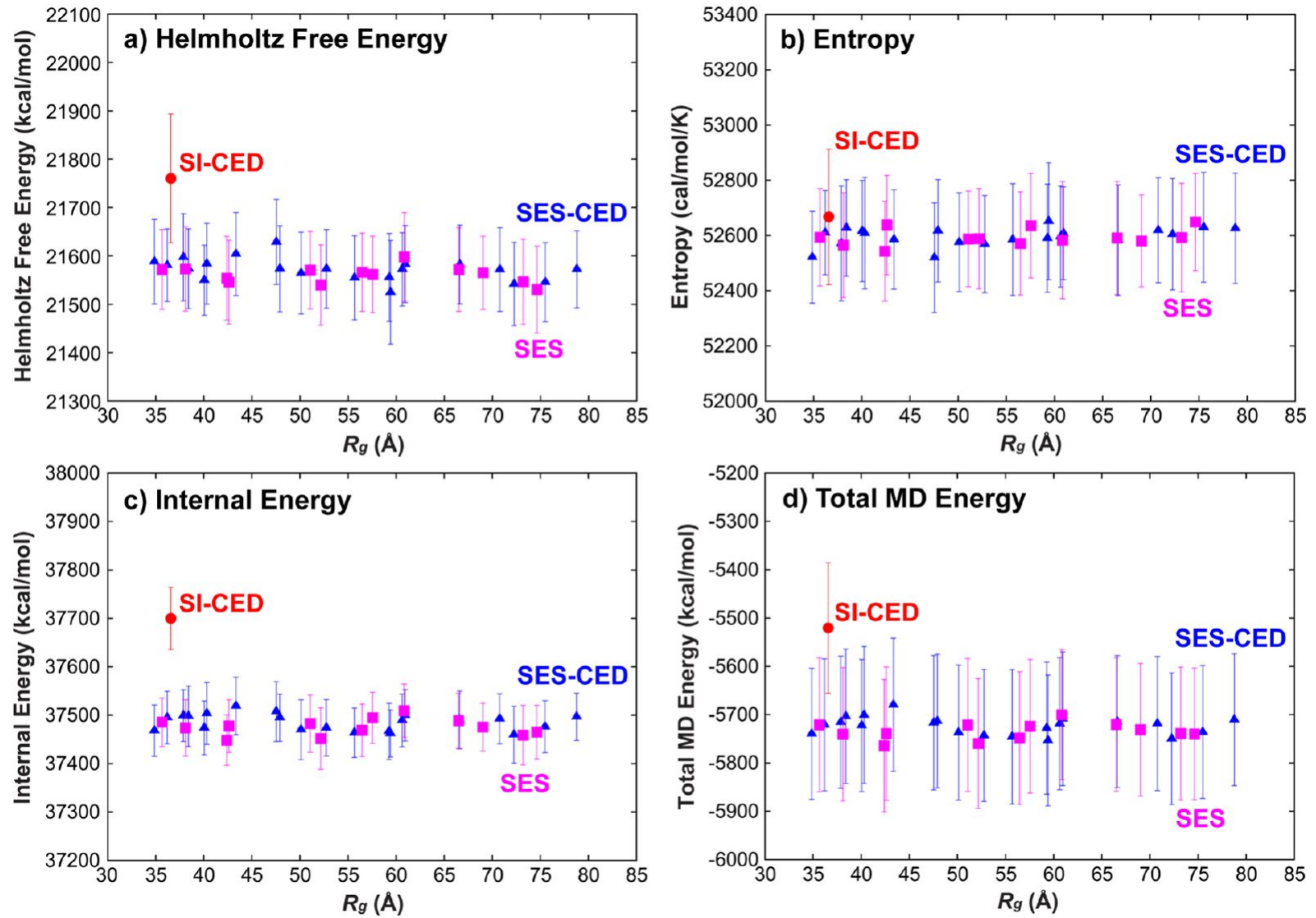

Figure 9. Thermodynamics calculated by the 2PT method over the last 1 ns of NVT dynamics. (a) Helmholtz free energies, (b) entropies, (c) internal energies, and (d) total MD energies. Red circles show the results from the solvent-included CED (SI-CED) calculations. The blue triangles show the results from using the SES-CED model (with $f=0.3)$ starting the simulation from the half of the experimental value $\left(0.5 \rho^{\exp }\right)$ as the initial density, followed by density and temperature annealing to near the experimental density $\left(\rho^{\exp }\right)$. The magenta squares show the SES results starting the simulation from the experimental density $\left(\rho^{\text {exp }}\right)$ as the initial density. The error bars are the standard deviation of each energetic property over 100 points. The standard deviation of $R_{\mathrm{g}}$ ( $x$-axis error bar) is smaller than the marker size for all samples. The MD energy here is the total energy (sum of the kinetic energy and potential energy) from the last $1 \mathrm{~ns}$ of NVT dynamics, $-5520.58 \pm 135.39 \mathrm{kcal} / \mathrm{mol}$ (SI-CED), $-5722.74 \pm 137.72 \mathrm{kcal} / \mathrm{mol}$ (SES$\mathrm{CED}$ ), and $-5734.67 \pm 137.23 \mathrm{kcal} / \mathrm{mol}(\mathrm{SES})$, without zero point energy and thermal corrections. These results show that the SES values are equivalent to the SES-CED values, indicating no extra advantage to the CED process for this system. In contrast, the simple SI-CED leads to significantly higher energies. Note however that the entropy is similar to that from SES, indicating that the main error in SI-CED is strain in the conformation that has not been fully relaxed by the CED process, leading to $\sim 200 \mathrm{kcal} / \mathrm{mol}$ of extra strain energy $(0.45 \mathrm{kcal} / \mathrm{mol}$ per monomer).

$0.01 \mathrm{~g} / \mathrm{cm}^{3}$ leads to R-coil PEG structures exhibiting a wide size range, $\Delta R_{\mathrm{g}} \approx 110 \AA$, with the most probable value of $R_{\mathrm{g}}=65.58$ $\AA$. Therefore, we performed NVT MD using $f=0.3$ on the single isolated polymer chain in a $63.52 \times 63.52 \times 63.52 \AA^{3}$ simulation box, leading to a density of $\rho=0.13 \mathrm{~g} / \mathrm{cm}^{3}$ (if we insert $75 \mathrm{wt} \%$ water, then the total density should be $0.52 \mathrm{~g} / \mathrm{cm}^{3}$, which is half of the density expected from experiment $\rho^{\exp }=1.04 \mathrm{~g} / \mathrm{cm}^{3}$ ). This procedure is to obtain a wide range of the initial ensemble of polymer chain conformations at half of the target density. Figure $7 \mathrm{~b}$ shows that in the case of $f=0.3$, compared with the case of a very low density system $\left(140 \times 140 \times 140 \AA^{3}\right)$ (blue solid line), the range of $R_{\mathrm{g}}$ of the chains from the $63.52 \times 63.52 \times 63.52 \AA^{3}$ simulation box decreased due to interactions with the image chains at higher density.

4.4. SES-CED Method. The steps of the SES-CED method are as follows:

(1) Grow the polymer chain using standard MC.

(2) Carry out MD calculations on the isolated chain using an appropriate range of the nonbonding scaling factor $f$ and a box of sufficiently low density to avoid chain-chain interactions with its periodic image. For our PEG-20 kDa system aimed at 25 wt \% aqueous solution, we started with a $63.52 \times 63.52 \times 63.52 \AA^{3}$ simulation box, which would lead to a density of $\rho=0.52 \mathrm{~g} / \mathrm{cm}^{3}$ after adding water (assuming that 75 wt \% water is included), which is $50 \%$ of the density measured from experiment, ${ }^{48} \rho^{\exp }=$ $1.04 \mathrm{~g} / \mathrm{cm}^{3}$, for $25 \mathrm{wt} \%$ PEG. The actual density before adding water is $0.13 \mathrm{~g} / \mathrm{cm}^{3}$. This procedure was described above in section 4.3. From the distribution in Figure $7 \mathrm{~b}$ for $f=0.3$ and $\rho=$ $0.13 \mathrm{~g} / \mathrm{cm}^{3}$, we selected 21 different PEG chains spaced evenly over the range of $R_{\mathrm{g}}=33.80-95.68 \AA$ for use in the SES-CED procedure.

(3) Using the equilibrated polymer chains from step 2, introduce water molecules into each simulation cell but change $f$ back to 1 because we now account for explicit solvation. To find favorable locations for the addition of water molecules without perturbing the polymer conformation, we carried out grand canonical Monte Carlo (GCMC) simulations while fixing the final number of water loading to the target value (3339 for the 25 wt $\%$ case).

(4) Then, starting with the chain-water distributions from step 3, compress the simulation cell gradually (uniformly over $\sim 3$ ns) from half of the expected density $\left(0.5 \rho^{\exp }=0.52 \mathrm{~g} / \mathrm{cm}^{3}\right)$ up to 1.1 times the expected density $\left(1.1 \rho^{\exp }=1.14 \mathrm{~g} / \mathrm{cm}^{3}\right)$ while carrying out temperature annealing between 300 and $706 \mathrm{~K}$ 

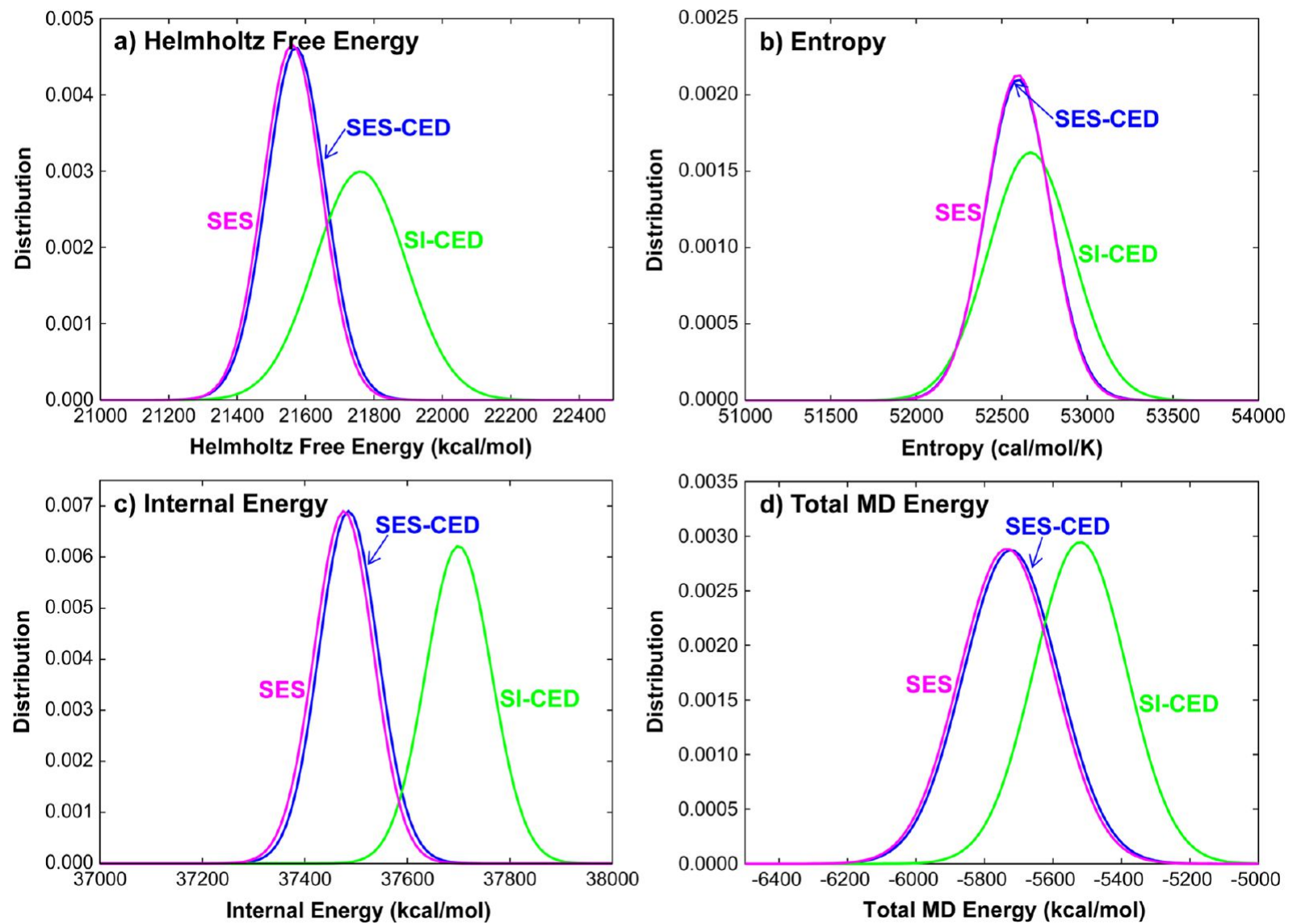

Figure 10. Gaussian distribution for each energetic property, (a) Helmholtz free energies $(A)$, (b) entropies $(S)$, (c) internal energies $(E)$, and (d) total MD energies for the cases of SI-CED (green line), SES-CED (blue line), and SES (magenta line). These figures show that the SES/SES-CED method leads to well-equilibrated PEG conformations in a 25 wt \% PEG aqueous solution, compared to the SI-CED method. Considering all 21 of the SES-CED configurations at the ensemble, we calculated $A=21572.37 \pm 84.51 \mathrm{kcal} / \mathrm{mol}, S=52595.63 \pm 188.42 \mathrm{cal} / \mathrm{mol} / \mathrm{K}$, and $U=37485.77 \pm 55.74 \mathrm{kcal} / \mathrm{mol}$. Considering all 13 of the SES configurations at the ensemble, we calculated $A=21561.05 \pm 84.37 \mathrm{kcal} / \mathrm{mol}, S=52593.32 \pm 185.63 \mathrm{cal} / \mathrm{mol} / \mathrm{K}$, and $U=$ $37475.39 \pm 55.68 \mathrm{kcal} / \mathrm{mol}$.

(approximately twice the Flory temperature; $353 \mathrm{~K}^{39}$ ). This was done for 5 cycles.

(5) Starting with the chain-water distributions with the density of $1.1 \rho^{\text {exp }}$ from step 4 , carry out NPT MD at 1 atm and $300 \mathrm{~K}$ for $4-15 \mathrm{~ns}$ to determine the equilibrium density $\left(\rho^{\text {eq. }}\right)$. The full SES-CED procedure leads to final densities of the 21 samples (with explicit waters) ranging from $\rho^{\mathrm{eq}}=1.0555$ to $1.0571 \mathrm{~g} / \mathrm{cm}^{3}$, which agrees with the experimental density ${ }^{48}$ $\left(\rho^{\exp }\right)$ of $1.0417 \mathrm{~g} / \mathrm{cm}^{3}$ within $1 \%$ for $25 \%$ PEG. This provides confidence that the water-PEG interaction is well described by our new FF and that the SES-CED procedure leads to wellequilibrated structures.

(6) At the predicted equilibrium density from step 5, perform NVT MD for 10-20 ns. The $1 \mathrm{~ns}$ trajectories are used for the thermodynamic and structural analyses by using $2 \mathrm{PT}$ method. ${ }^{29}$ We performed thermodynamic analyses on the sampled polymer conformers immersed in solvent to calculate the thermodynamic preference of the polymer configuration in solvent.

4.5. Thermodynamic Analysis Using the 2PT Density of States (DoS) Method. To determine the thermodynamic properties of the PEG-water systems, we used 2PT theory, which partitions the vibrational density of states (DoS) from the Fourier transform of the velocity autocorrelation function into diffusional contributions (leading to $\operatorname{DoS}(\nu) \neq 0$ at $\nu=0$ ) and a solid-like vibrational contribution (leading to $\operatorname{DoS}(\nu)=0$ at $\nu=$ $0)$.
The trajectories from the last $1 \mathrm{~ns} N V T$ dynamics were used for the thermodynamic and structural analyses. We used the $2 \mathrm{PT}$ method $^{29}$ to predict the thermodynamic properties of the polymer conformations immersed in solvent. This is used to calculate the thermodynamically preferred polymer configuration in solvent. Because $2 \mathrm{PT}$ requires analysis only over a period of 20 ps to get an accurate free energy and entropy, we restarted the trajectories every 10 ps over the last $1 \mathrm{~ns}$ of NVT and did the 20 ps $2 \mathrm{PT}$ analysis 100 times to provide both thermodynamic quantities and their fluctuations.

Figure 8 shows the DoS of PEG for a representative case, $R_{\mathrm{g}}=$ $55.67 \AA$. [Figures S10-S12 (SI) show the full DoS and the partition into PEG and water parts for three representative cases, $R_{\mathrm{g}}=34.86,55.67$, and $78.77 \AA$..] This DoS leads to a partition function from which we calculate the Helmholtz free energy $(A)$, entropy $(S)$, and internal energy $(E)$ for the 21 independent samples.

This leads to the results shown in Figure 9a-c. Because the dynamics of PEG in the solvated system leads to only marginal changes in its chain conformation during the $1 \mathrm{~ns} \mathrm{MD}$ simulation, we regard each calculated thermodynamic quantity as the local value for the given PEG conformation, while we consider that the Boltzmann average over all 21 conformations provides the thermodynamic quantities for the equilibrium polymer-water system. This leads to an average over the entire 


\section{Final PEG conformations after SES-CED}

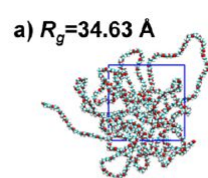

d) $R_{g}=38.11 \mathrm{~A}$
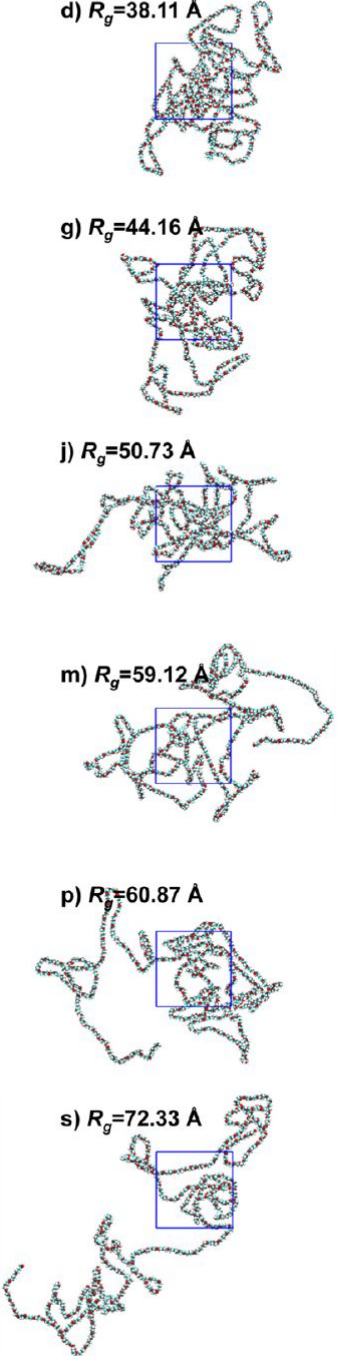
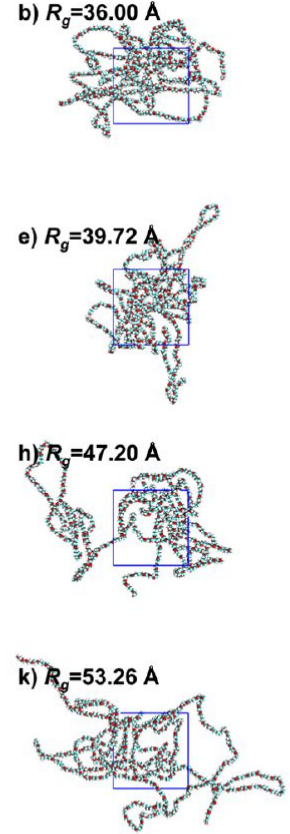

n) $R_{g}=59.22 \mathrm{~A}$
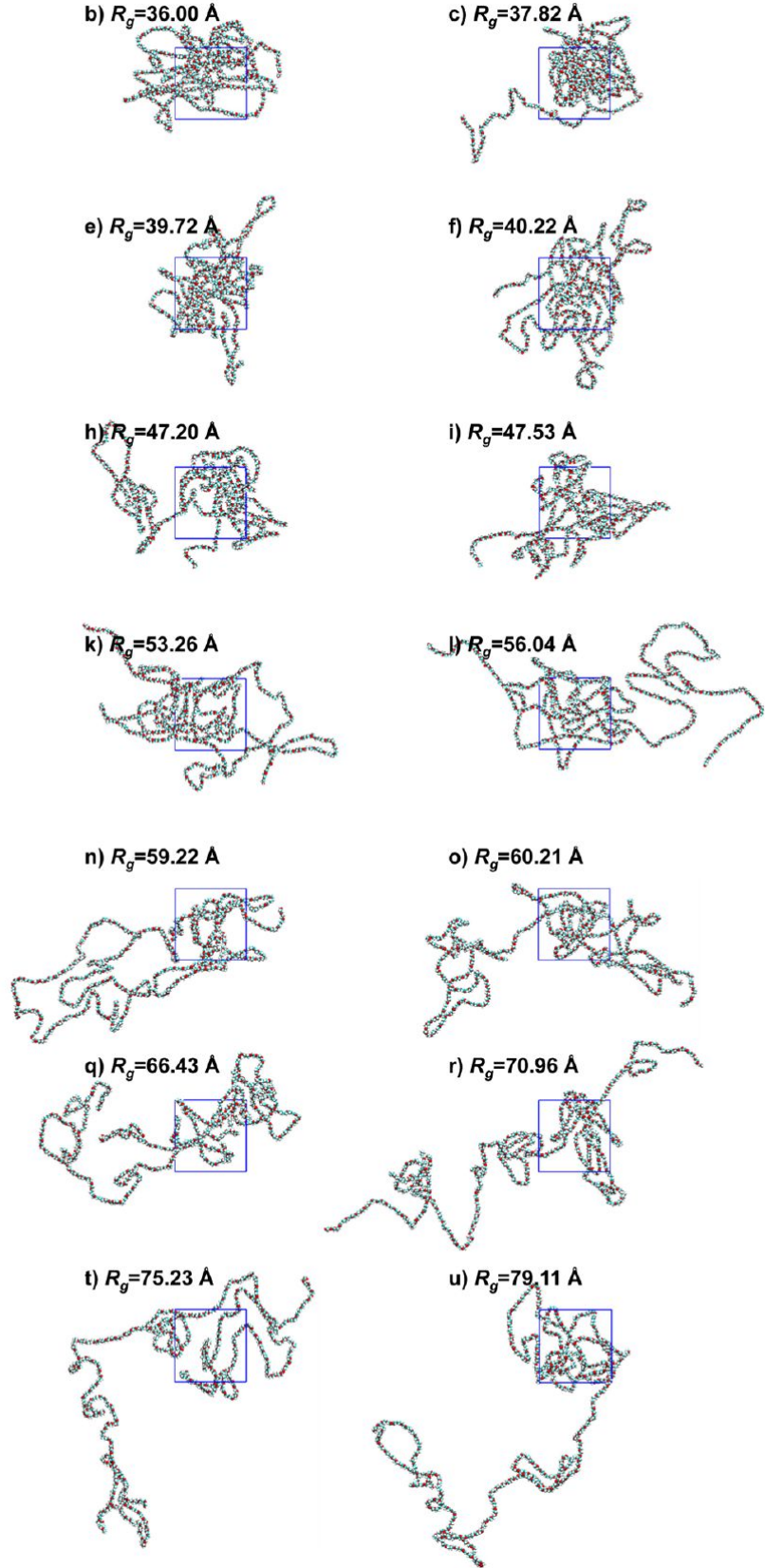

o) $R_{g}=60.21 \mathrm{~A}$

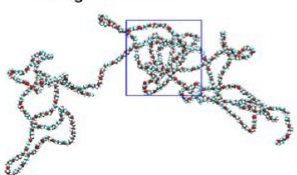

Figure 11. The final chain conformations of a PEG in aqueous solutions with 25 wt \% PEG for the 21 cases of SES-CED. The final values of $R_{\mathrm{g}}$ are shown in the figure. White, red, and green atoms are hydrogen, oxygen, and carbon atoms, respectively. The periodic box is shown as a blue solid line. For all cases, the final densities range from 1.0555 to $1.0571 \mathrm{~g} / \mathrm{cm}^{3}$, comparable with the experimental density ${ }^{48}$ of $1.0417 \mathrm{~g} / \mathrm{cm}^{3}$. The initial values of $R_{\mathrm{g}}$ were (a) 41.95, (b) 33.80, (c) 38.81, (d) 36.03, (e) 48.23, (f) 45.03, (g) 51.28, (h) 63.77, (i) 54.40, (j) 60.66, (k) 57.56, (1) 73.14, (m) 79.34, (n) 66.82, (o) 76.20, (p) 70.00, (q) 82.48, (r) 85.01, (s) 90.59, (t) 88.90, and (u) $95.68 \AA$.

phase space from separate integrations over the subvolume of each nearly fixed PEG conformation.

Figure $9 a-c$ shows that all 21 samples obtained from SESCED lead to very similar free energies (with standard deviations of $\sigma_{A}=22.92 \mathrm{kcal} / \mathrm{mol} / \mathrm{cell}, \sigma_{E}=17.05 \mathrm{kcal} / \mathrm{mol} / \mathrm{cell}$, and $\sigma_{S}=$ $32.94 \mathrm{cal} / \mathrm{mol} / \mathrm{K} / \mathrm{cell}$ ) with respect to their statistical fluctuations due to the solvent molecules.

Figure 10 shows the result of Boltzmann weighting the distributions of energetic properties for each of the 21 cases in Figure 9 to obtain the thermodynamic average over the whole ensemble. This shows that the Helmholtz free energy from SESCED is substantially more stable than that from SI-CED.
The similarity of the energetics $(\Delta A \approx 104 \mathrm{kcal} / \mathrm{mol} / \mathrm{cell}$ compared with a total average $A=21572.37 \mathrm{kcal} / \mathrm{mol} / \mathrm{cell})$ for the large range of polymer conformations $\left(\Delta R_{\mathrm{g}} \approx 44 \AA\right)$ suggests that the conformational dynamics of the PEG in this semidilute regime $(25 \mathrm{wt} \%)$ is similar to the polymer dynamics in a $\theta$ solvent. ${ }^{49}$ That is, the characteristics of the PEG conformation are determined by the short-range properties of the polymer backbone.

4.6. Analysis of Results. Figure 9d shows that all 21 PEG structures lead to nearly equal total $\mathrm{MD}$ energies with a range from -5753.39 to $-5679.58 \mathrm{kcal} / \mathrm{mol}$, where the fluctuations within each system are $\pm 137.72 \mathrm{kcal} / \mathrm{mol}$. This gives us 
Final PEG conformations after SES
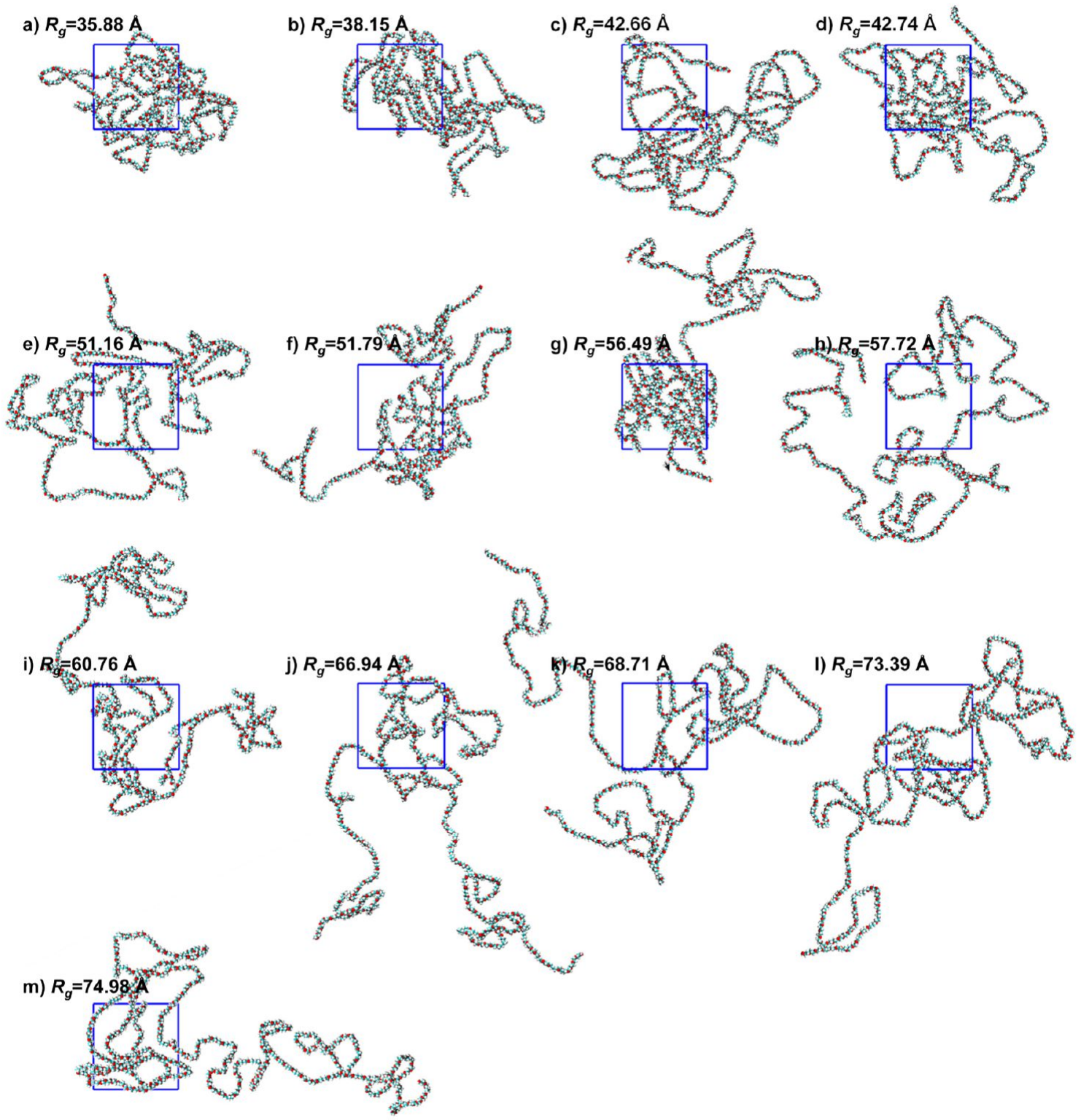

Figure 12. The final chain conformations of a PEG in aqueous solutions with $25 \mathrm{wt} \% \mathrm{PEG}$ for the 13 cases of SES. The final values of $R_{\mathrm{g}}$ are shown in the figure. White, red, and green atoms are hydrogen, oxygen, and carbon atoms, respectively. The periodic box is shown as a blue solid line. For all cases, the final densities range from 1.0557 to $1.0573 \mathrm{~g} / \mathrm{cm}^{3}$, which compare with the experimental density of $1.0417 \mathrm{~g} / \mathrm{cm}^{3}$. The initial values of $R_{\mathrm{g}}$ were $(\mathrm{a}) 30.35$, (b) 35.77, (c) 42.87, (d) 39.38, (e) 46.38, (f) 49.98, (g) 53.58, (h) 57.06, (i) 60.68, (j) 64.24, (k) 67.74, (l) 71.11, and (m) 72.30 ̊.

confidence that the procedure leads to well-equilibrated chainsolvent systems so that this range of sizes is expected to occur over the time scales of the experiment.

In contrast, the SI-CED method (red dot in Figure 9d) leads to a MD energy higher by $\sim 200 \mathrm{kcal} / \mathrm{mol}$ and to an $R_{\mathrm{g}}$ that is small compared to that of the range of structures from SES-CED.

We emphasize that the introduction of SES dynamics without explicit water molecules in steps 1 and 2 is crucial to achieve the wide range of polymer chain conformations within a short $(\sim 40$ ns) MD simulation time scale. This is because the conformational dynamics of the PEG chain in explicit water solvent changes only very slowly, with a standard deviation in $R_{\mathrm{g}}$ of $<0.60$ Å within $36 \mathrm{~ns}$ of $\mathrm{MD}$.

The final 21 PEG chain configurations of the equilibrated PEG-water system with 25 wt \% PEG chains sample a large range of PEG conformations with $R_{\mathrm{g}}$ varying from 34.86 to 78.77 $\AA$ A. Figure 11 shows the final chain conformations for the 21 cases of SES-CED.

On the basis of the Boltzmann distribution of free energies predicted for the 21 cases, we re-evaluated the $R_{\mathrm{g}}$, leading to $R_{\mathrm{g}}=$ $53.98 \AA$, which is the final thermodynamically averaged size from the SES-CED method for 25 wt \% PEG in water at $300 \mathrm{~K}$. These results are quite different than the result from SI-CED, which leads to $R_{\mathrm{g}}=36.57 \AA$, but are comparable to $R_{\mathrm{g}}=65 \AA$ from viscosity experiments ${ }^{50}$ in the dilute limit.

To interpret this $R_{\mathrm{g}}$ using the freely jointed polymer (Gaussian random coil) model would require a segment length of $l=13.16$ with $N=147$ segments to preserve the total length, leading to $\left\langle R_{\mathrm{g}}{ }^{2}\right\rangle^{1 / 2}=l(N / 6)^{1 / 2}=65.14 \AA$. 
This suggests that the ether oxygen atoms serve as the joints (with the rigid $\mathrm{O}-\mathrm{CH}_{2}-\mathrm{CH}_{2}-\mathrm{O}$ units). This leads to $l=4.251$ $\AA$, which, with $N=455$, leads to $\left\langle R_{\mathrm{g}}{ }^{2}\right\rangle^{1 / 2}=37.02 \AA$. This suggests that we use the freely rotating chain in which the $\mathrm{C}-\mathrm{O}-\mathrm{C}$ angle is fixed at the minimum energy value of $113.7^{\circ}$. This leads to $\left\langle R_{\mathrm{g}}{ }^{2}\right\rangle^{1 / 2}=37.02[(1+\cos \theta) /(1-\cos \theta)]^{1 / 2}=56.68 \AA$, where $\theta=$ $180-113.7=66.3^{\circ}$, which is in excellent agreement with the simulations.

\section{SES METHOD}

Our analysis on the results from the SES-CED calculations suggests that it might not be necessary to start with the half density cell. This leads to the SES method in which we start with the simulation box of $50.42 \times 50.42 \times 50.42 \AA^{3}$, leading to an initial density of $\rho=1.04 \mathrm{~g} / \mathrm{cm}^{3}=\rho^{\exp }$ (after including water molecules) rather than $0.5 \rho^{\exp }$ as in the previous SES-CED method. From the distribution obtained in the box with $\rho^{\exp }$, we selected 13 different PEG chains spaced evenly for use in this new SES procedure. By modifying step 2 of SES-CED in this way, we excluded the compression annealing from the SES-CED (step 4) and used only the NPT dynamics (step 5) to adjust the final simulation box size to have the optimal density. Because compression annealing of the simulation box is not involved here, we are able to transfer the PEG chain sampled from SES dynamics into the explicit solvation box. The equilibrated final structures are shown in Figure 12. The final densities of the 13 samples (with explicit waters) range from 1.0557 to $1.0573 \mathrm{~g} /$ $\mathrm{cm}^{3}$, which are within $1 \%$ of the experimental density ${ }^{48}$ of 1.0417 $\mathrm{g} / \mathrm{cm}^{3}$.

The trajectories from the last $1 \mathrm{~ns} N V T$ dynamics are used for the thermodynamic and structural analyses. Using the $2 \mathrm{PT}$ method, we perform thermodynamic analyses on the sampled polymer conformers immersed in solvent to calculate the thermodynamic preference of the polymer configuration in solvent. Figure 9 confirms that these 13 new structures have a range of energies and sizes similar to those of the 21 structures from the SES-CED procedure.

Calculating the Helmholtz free energies based on a standard Boltzmann distribution for the 13 cases leads to the Helmholtz free energy distribution in Figure 10a, which is slightly more stable than that for SES-CED. Using this Boltzmann-weighted distribution, we calculate $R_{\mathrm{g}}=55.48 \AA$ for 25 wt \% PEG, which is similar to the value of $R_{\mathrm{g}}(53.98 \AA$ ) calculated from SES-CED. Thus, the SES method lead to a broad range of well-equilibrated PEG conformations in water, in contrast to SI-CED, which leads to only one size of PEG conformation and a much higher energy (see Figures 9 and 10).

The optimum $R_{\mathrm{g}}$ from SES compares well with experimental ensemble-averaged $R_{\mathrm{g}}$ of PEG conformations in the dilute concentration regime; $R_{\mathrm{g}}=57.11-69.17 \AA$ from SLS measurements, ${ }^{12,15,51}$ and $R_{\mathrm{g}}=65 \AA$ from viscosity measurements. ${ }^{50}$

This SES calculation indicates that properly equilibrated chain-water structures can be constructed directly at the target density without the density annealing steps. We expect that this SES procedure can be useful in future simulations.

\section{CONCLUSIONS}

We present and validate here the new SES method for sampling a wide range of polymer chain sizes for a high-molecular-weight (20 kDa) PEG chain dissolved in water for polymer concentrations of $25 \mathrm{wt} \%$.
Our detailed thermodynamic analysis shows that the polymer configurations are determined mostly by short-range properties of the polymer backbone allowing substantial size variations in the PEG chains $\left(\Delta R_{\mathrm{g}}=43.91 \AA\right.$ from SES-CED and $\Delta R_{\mathrm{g}}=38.98$ $\AA$ from SES) for solvation in the semidilute regime of $25 \mathrm{wt} \%$. This is because the stiffness of the PEG backbone opens sufficient space for water molecules to form a strong first solvation shell (via a short-range interaction), stabilizing the polymer independently.

We expect that the SES method will be useful to investigate comprehensively the solvent-polymer interaction with $\mathrm{MD}$ simulations, as illustrated with this study of PEG-water systems. Such MD studies should provide atomistic insights into PEGwater interactions in the semidilute regime (where experimental characterization on the structure is almost impossible), which is directly related to the nonlinear increase of osmotic behavior of PEG.

\section{ASSOCIATED CONTENT}

\section{S Supporting Information}

The comparison between QM calculation results and the results calculated by our optimum FF is shown in Figures S1 and S2, the optimum FF parameters are listed in Table S1, the partial charge distributions of a PEG-20 kDa are shown in Table S2, the $R_{\mathrm{g}}$ variation with time for a scaling factor ranging from 0.1 to 1.0 and the temperature ranging from 100 to $1200 \mathrm{~K}$ is illustrated in Figure S3, the snapshots of the conformations with time and temperature for $f=0.1,0.5$, and 0.7 are shown in Figures S4-S6, the determination of the $\theta$ temperature for each scaling factor is explained in Figure $\mathrm{S} 7$, the scaled standard deviations of $R_{\mathrm{g}}$ at the highest temperature for each scaling factor are listed in Table S3, the scaled standard deviation of $R_{\mathrm{g}}$ for the ideal Gaussian chains with a different number of monomers or polymer chains is shown in Table S4, the $R_{\mathrm{g}}$ distribution of random coils sampled with $f=$ 0.3 as a function of temperature is illustrated in Figure $S 8$, the $R_{\mathrm{g}}$ distributions of random coils sampled with $f=0.1$ or 0.3 are shown in Figure S9, and the density of states for three representative cases $R_{\mathrm{g}}=34.86,55.67$, and $78.77 \AA$ are shown in Figures S10-S12. This material is available free of charge via the Internet at http://pubs.acs.org.

\section{AUTHOR INFORMATION}

\section{Corresponding Author}

*E-mail: wag@wag.caltech.edu (W.A.G.); linus16@kaist.ac.kr (H.K.).

\section{Notes}

The authors declare no competing financial interest.

\section{ACKNOWLEDGMENTS}

This research was supported by World Class University Program (R31-2008-000-10055-0) and the Integrated Water Technology (IWT) Project (2012M1A2A2026588) funded by the Ministry of Education, Science and Technology through the National Research Foundation of Korea. We thank the support from the Energy, Environment, Water, and Sustainability Initiative funding from the Korea Advanced Institute of Science and Technology (KAIST).

\section{REFERENCES}

(1) Bekiranov, S.; Bruinsma, R.; Pincus, P. Phys. Rev. E 1997, 55, 577585 .

(2) Harris, J. M. Poly(Ethylene Glycol) Chemistry: Biotechnical and Biomedical Applications; Plenum: New York, 1992; p 385. 
(3) Veronese, F. M.; Pasut, G. Drug Discovery Today 2005, 10, 14511458

(4) Huang, Y. Y.; Chung, T. W.; Tzeng, T. W. Int. J. Pharm. 1999, 182, 93-100.

(5) Molineux, G. Cancer Treat. Rev. 2002, 28, 13-16.

(6) Knop, K.; Hoogenboom, R.; Fischer, D.; Schubert, U. S. Angew. Chem., Int. Ed. 2010, 49, 6288-6308.

(7) Liu, Z.; Robinson, J. T.; Sun, X. M.; Dai, H. J. J. Am. Chem. Soc. 2008, 130, 10876-10877.

(8) Le Coz, C. J.; Heid, E. Contact Dermatitis 2001, 44, 308-319.

(9) Fruijtier-Polloth, C. Toxicology 2005, 214, 1-38.

(10) Ye, Y.; Rick, J.; Hwang, B. Polymers 2012, 4, 913-963.

(11) Devanand, K.; Selser, J. C. Nature 1990, 343, 739-741.

(12) Polverari, M.; van de Ven, T. G. M. J. Phys. Chem. 1996, 100, 13687-13695.

(13) Ho, D. L.; Hammouda, B.; Kline, S. R. J. Polym. Sci., Part B: Polym. Phys. 2003, 41, 135-138.

(14) Polik, W. F.; Burchard, W. Macromolecules 1983, 16, 978-982.

(15) Kawaguchi, S.; Imai, G.; Suzuki, J.; Miyahara, A.; Kitano, T. Polymer 1997, 38, 2885-2891.

(16) Maeda, Y.; Tsukida, N.; Kitano, H.; Terada, T.; Yamanaka, J. J. Phys. Chem. 1993, 97, 13903-13906.

(17) Maxfield, J.; Shepherd, I. W. Polymer 1975, 16, 505-509.

(18) Derkaoui, N.; Said, S.; Grohens, Y.; Olier, R.; Privat, M. J. Colloid Interface Sci. 2007, 305, 330-338.

(19) Liu, K.; Parsons, J. L. Macromolecules 1969, 2, 529-533.

(20) Faraone, A.; Magazu, S.; Maisano, G.; Migliardo, P.; Tettamanti, E.; Villari, V. J. Chem. Phys. 1999, 110, 1801-1806.

(21) Hammouda, B.; Ho, D.; Kline, S. Macromolecules 2002, 35, 85788585 .

(22) Hammouda, B.; Ho, D. L.; Kline, S. Macromolecules 2004, 37, 6932-6937.

(23) Kozer, N.; Kuttner, Y. Y.; Haran, G.; Schreiber, G. Biophys. J. 2007, 92, 2139-2149.

(24) Tasaki, K. J. Am. Chem. Soc. 1996, 118, 8459-8469.

(25) Smith, G. D.; Bedrov, D.; Borodin, O. Phys. Rev. Lett. 2000, 85, $5583-5586$.

(26) Smith, G. D.; Bedrov, D.; Borodin, O. J. Am. Chem. Soc. 2000, 122, 9548-9549.

(27) Borodin, O.; Bedrov, D.; Smith, G. D. Macromolecules 2001, 34, $5687-5693$.

(28) Boils, D.; Hair, M. L. J. Colloid Interface Sci. 1993, 157, 19-23.

(29) Lin, S. T.; Blanco, M.; Goddard, W. A., III. J. Chem. Phys. 2003, 119, 11792-11805.

(30) Mayo, S. L.; Olafson, B. D.; Goddard, W. A., III. J. Phys. Chem. 1990, 94, 8897-8909.

(31) Zhao, Y.; Truhlar, D. G. Theor. Chem. Acc. 2008, 120, 215-241.

(32) Dunning, T. H. J. Chem. Phys. 1989, 90, 1007-1023.

(33) Jaguar, version 7.6; Schrödinger, LLC: New York, 2009.

(34) Levitt, M.; Hirshberg, M.; Sharon, R.; Laidig, K. E.; Daggett, V. J. Phys. Chem. B 1997, 101, 5051-5061.

(35) Plimpton, S. J. Comput. Phys. 1995, 117, 1-19.

(36) Sadanobu, J.; Goddard, W. A., III. Fluid Phase Equilib. 1998, 144, 415-425.

(37) Sadanobu, J.; Goddard, W. A., III. J. Chem. Phys. 1997, 106, 67226729.

(38) Belmares, M.; Blanco, M.; Goddard, W. A., III; Ross, R. B.; Caldwell, G.; Chou, S. H.; Pham, J.; Olofson, P. M.; Thomas, C. J. Comput. Chem. 2004, 25, 1814-1826.

(39) Venohr, H.; Fraaije, V.; Strunk, H.; Borchard, W. Eur. Polym. J. 1998, 34, 723-732.

(40) Jaramillo-Botero, A.; Blanco, M.; Li, Y. Y.; McGuinness, G.; Goddard, W. A., III. J. Comput. Theor. Nanosci. 2010, 7, 1238-1256.

(41) Henz, B. J.; Chung, P. W.; Andzelm, J. W.; Chantawansri, T. L.; Lenhart, J. L.; Beyer, F. L. Langmuir 2011, 27, 7836-7842.

(42) Rai, N.; Wagner, A. J.; Ross, R. B.; Siepmann, J. I. J. Chem. Theory Comput. 2008, 4, 136-144.

(43) Jang, S. S.; Molinero, V.; Cagin, T.; Goddard, W. A., III. J. Phys. Chem. B 2004, 108, 3149-3157.
(44) Jang, S. S.; Goddard, W. A., III. J. Phys. Chem. C 2007, 111, 27592769.

(45) Jeon, J.; Kim, H.; Goddard, W. A., III; Pascal, T. A.; Lee, G. I.; Kang, J. K. J. Phys. Chem. Lett. 2012, 3, 556-559.

(46) Jang, S. S.; Molinero, V.; Cagin, T.; Goddard, W. A., III. Solid State Ionics 2004, 175, 805-808.

(47) Jang, S. S.; Cagin, T.; Goddard, W. A., III. J. Chem. Phys. 2003, 119, $1843-1854$.

(48) Cruz, R. D.; Martins, R. J.; Cardoso, M. J. E. D.; Barcia, O. E. J. Solution Chem. 2009, 38, 957-981.

(49) Adam, M.; Delsanti, M. Macromolecules 1985, 18, 1760-1770.

(50) Kato, T.; Nakamura, K.; Kawaguchi, M.; Takahashi, A. Polym. J. 1981, 13, 1037-1043.

(51) Devanand, K.; Selser, J. C. Macromolecules 1991, 24, 5943-5947. 